\title{
\begin{tabular}{l|l} 
Received: 2010.03 .02 & Current medical research with the application of \\
Accepted: & 2010.09 .27
\end{tabular} Publishet: 2011.05 .01 coupled techniques with mass spectrometry
}

\section{Joanna Kałużna-Czaplińska}

Department of Chemistry, Institute of General and Ecological Chemistry, Technical University of Lodz, Lodz, Poland

Source of support: Polish Ministry of Science and Higher Education (No. NN 204 316234)

\section{Summary}

The most effective methods of analysis of organic compounds in biological fluids are coupled chromatographic techniques. Capillary gas chromatography/mass spectrometry (GC-MS) allows the most efficient separation, identification and quantification of volatile metabolites in biological fluids. Liquid chromatography-mass spectrometry (LC-MS) is especially suitable for the analysis of non-volatile and/or thermally unstable compounds. A major drawback of liquid chromatography-mass spectrometry is that no standard spectral libraries such as NIST and Wiley for GC-MS are available to facilitate the identification of unknown compounds. Moreover, the identification of potential new compounds, especially new biomarkers in LC-MS, is much more challenging than in GC-MS. Capillary electrophoresis coupled with mass spectrometry (CE-MS) has been widely used to characterize metabolomes. Capillary electrophoresis is a powerful technique for the separation of charged metabolites, offering high analyte resolution. The advantages of CE-MS are applicability for hydrophilic metabolites, robust separation efficiency and short duration of analysis.

This review provides an overview of current chromatographic methods - gas chromatography mass spectrometry, liquid chromatography - mass spectrometry and capillary electrophoresis-mass spectrometry - and their applications in current medical research. The focus is on the description of metabonomics research, strategies for biomarkers identification, medical diagnoses of diseases and research of drugs.

key words: $\quad$ gas chromatography • liquid chromatography • capillary electrophoresis • mass spectrometry - medical research

Full-text PDF:

http://www.medscimonit.com/fulltxt.php?ICID=881756

Word count:

3470

Tables:

2

Figures:

1

43 


\section{BACKGROUND}

Current medical research (research connected with the monitoring of the concentration of metabolites in body fluids, tissue or breathing, as well as research looking for markers necessary in correct diagnosing and interference of many diseases) would be impossible without modern instrument techniques, especially those based on the use of chromatography and electrophoresis coupled with mass spectrometry. The techniques most often used in clinical research are as follows: gas chromatography - mass spectrometry (GC-MS), gas chromatography - tandem mass spectrometry (GC-MS/MS), liquid chromatography - mass spectrometry (LC-MS), liquid chromatography - tandem mass spectrometry (LC-MS/MS), ultra-performance liquid chromatography - mass spectrometry (UPLC-MS), nuclear magnetic resonance - mass spectrometry (LC-NMR-MS) and capillary electrophoresis - mass spectrometry (CE-MS). These techniques are used not only in diagnosing and early medical intervention, but also in research on pharmacokinetics and pharmacodynamics of drugs, metabolomics and metabolic profiling analysis. GC-MS is a hyphenated technique for the analysis of volatile and semi-volatile compounds, suitable for chemical derivatization in order to increase volatility and thermal stability of these compounds. The combination of gas chromatography with electron impact ionization MS (EI-MS) provides high-chromatographic metabolite resolution, analyte-specific detection, and quantification of

Table 1. Coupled techniques used in medical research and their characteristics $[1,3,11]$.

\begin{tabular}{cccc}
\hline Technique & Sensitivity & \multicolumn{2}{c}{ Throughput Comprehensiveness } \\
\hline GC-MS & High & High & High \\
\hline LC-MS & Medium & High & High \\
\hline CE-MS & High & Medium & High \\
\hline
\end{tabular}

metabolites, as well as the ability to identify unknown compounds. Electron ionization (EI) in GC-MS is quite reproducible and is less affected by ion suppression during ionization than are other ionization methods. The characteristic and constant fragmentation patterns for certain compounds provided by electron ionization facilitate structural identification through the search for spectral reference libraries such as NIST, EPA and Wiley mass spectral databases [1]. Unlike GC-MS, liquid chromatography-mass spectrometry (LC-MS) is especially suitable for the analysis of non-volatile and/or thermally unstable compounds.

Currently, a major drawback of LC-MS is that no standard spectral libraries such as NIST and Wiley for GC-MS are available to facilitate the identification of unknown compounds. Thus, the identification of potential new compounds, especially new biomarkers in LC-MS, is much more challenging than in GC-MS [2]. In recent years capillary electrophoresis coupled with mass spectrometry (CE-MS) has been widely used to characterize metabolomes. CE is a powerful technique for the separation of charged metabolites, offering high analyte resolution. The advantages of CE-MS are applicability for hydrophilic metabolites, robust separation efficiency and short duration of analysis. Tables 1 and 2 present general characteristics of coupled techniques used in medical research.

Current gas methods of chromatography-mass spectrometry, liquid chromatography - mass spectrometry and capillary electrophoresis-mass spectrometry and their current applications in medical research are summarized in this review.

\section{Coupled Techniques in Current Metabolomics and MEDICAL DiSEASE Diagnosis}

Metabolomics research currently plays the most significant role in clinical chemistry. The analyses of human metabolome or metabolites present in cells in the form of organic compounds (amino acids, organic acids, fatty acids, carbohydrates, vitamins and fats) are the subject of interest of many contemporary scientists. Techniques coupled with

Table 2. Comparison of analytical methods: GC-MS, LC-MS, CE-MS [1,2,11].

\begin{tabular}{clll}
\hline $\begin{array}{c}\text { Analysis } \\
\text { technique }\end{array}$ & \multicolumn{1}{c}{ Application } & \multicolumn{1}{c}{ Advantages } & Disadvantages \\
\hline GC-MS & $\begin{array}{l}\text { Separation, identification, } \\
\text { quantification of different compounds } \\
\text { (volatile and non-volatile) in a single } \\
\text { analysis. The type of analysis is defined } \\
\text { by switching the detection between } \\
\text { the SIM and SCAN modes }\end{array}$ & $\begin{array}{l}\text { High resolution, ideal to resolve } \\
\text { complex biological samples. Possible } \\
\text { simultaneous analysis of different } \\
\text { compounds classes }\end{array}$ & $\begin{array}{l}\text { Impossible analysis of thermolabile } \\
\text { compounds (metabolites). Non-volatile } \\
\text { metabolites must be derivatized before } \\
\text { analysis. Difficulties in identification } \\
\text { of unknown compounds after } \\
\text { derivatization }\end{array}$ \\
& $\begin{array}{l}\text { Separation, identification, } \\
\text { quantification of a very broad group } \\
\text { of compounds. Limited potential } \\
\text { in identification unless the MS/MS } \\
\text { technique is used }\end{array}$ & $\begin{array}{l}\text { High sensitivity, average to high } \\
\text { chromatographic resolution, } \\
\text { derivatization is unnecessary, possible } \\
\text { analysis of thermolabile compounds } \\
\text { (metabolites) }\end{array}$ & $\begin{array}{l}\text { A few restrictions on } \\
\text { LC eluents. De-salting may be needed. } \\
\text { Limited structural information. Matrix } \\
\text { effect }\end{array}$ \\
\hline LC-MS & $\begin{array}{l}\text { Separation, identification, } \\
\text { quantification of polar compounds, } \\
\text { using small sample volumes }\end{array}$ & $\begin{array}{l}\text { Useful for complex biological samples. } \\
\text { Small volumes. High resolution }\end{array}$ & $\begin{array}{l}\text { Complex methodology and } \\
\text { quantification. Buffer incompatibility. } \\
\text { Difficulties in interfacing. Further } \\
\text { development is necessary }\end{array}$ \\
\hline
\end{tabular}


chromatography and electrophoresis make it possible to observe changes in the concentrations of metabolic products in biological samples such as body fluids and tissue. The contents of metabolites in these matrices may exceed the estimated magnitude value of $10^{-7}-10^{-9}$. In the case of metabolomics, the GC-MS technique is described as a gold standard, although it is inconvenient for the non-volatile metabolites with high molecular mass [3]. The LC-MS instruments do not allow obtaining the high degree of reliable results possible with GC-MS; however, LC-MS instruments are approved in analyses of materials which are too volatile or thermally unstable. LC-MS is the method with higher potential applicability and easier preparation of samples before analysis. Coupling of chromatography with tandem mass spectrometry (GC-MS and LC-MS) improves the sensitivity of analysis and its specificity. These techniques are used in analyses of complex mixtures, making it possible to identify dozens of compounds in a sample [2]. In the case of CE-MS, separation of a compounds mixture may be controlled by the change of $\mathrm{pH}$. This technique is useful when other methods do yield repeatable results. CE-MS coupling results in a potential increase in sensitivity and specificity and enables potential identification of unknown compounds. This technique does not require any complicated preliminary preparations of samples for analysis [4].

\section{COUPLed TeChNiques in the Diagnosis of Metabolic DISORDERS}

Coupled techniques are applied in the diagnosis of metabolic disorders. Inborn Errors of Metabolism (IEM) are genetic disorders caused by alterations of a specific chemical reaction in the metabolism. Currently, there are about 400 defects related to pathology, and about 1 infant in 200 is affected. The pathogenesis of IEM can generally be attributed to the loss or gain of function of mutant proteins (usually an enzyme or transporter) [5,6]. A diagnostic procedure with an application of highly sensitive GC-MS analysis of urine metabolome is useful for screening and chemical diagnosis of IEM. Metabolite analysis can comprehensively detect enzyme dysfunction caused by a variety of abnormalities. Mutations may be uncommon or unknown. The lack of coenzymes or activators and the presence of posttranslational modification defects and subcellular localization of abnormalities are also reflected in the metabolome. This non-invasive and practical urine metabolome analysis, which uses urease pretreatment, partial adoption of stable isotope dilution, and GC-MS technique, can be applied in detection of more than 130 metabolic disorders, and can also detect an acquired abnormal metabolic profile. Many inborn errors of metabolism classified as organic acidurias, in which organic acids are present in urine and amino acids are present in metabolism disorders, can be discovered with the application of GC-MS [5-7]. For example, it enables recognition of the following diseases: methylmalonic aciduria, propionic acidemia, isovaleric aciduria, glutaric aciduria Type 1 and Type 2, urea cycle disorders, dicarboxylic aciduria, lactic aciduria, maple syrup urine disease, tyrosinemia, phenylketonuria, alkaptonuria, hypermethioninemia, cystinuria, galactosemia, and mitochondrial fatty acid oxidation disorders $[8,9]$. Apart from the analysis of urine, the diagnosis of organic acids disorders may be made prenatally through the analysis of amniotic fluid. In this case, the GC-MS technique enables a 100\% accuracy of diagnosis, which is confirmed later by postnatal examination of urine. This helps to avoid accidents of sudden and unexpected infant death due to organic acidurias [8].

LC-MS/MS is a second, powerful tool for the study of metabolic disorders. The analysis of acylcarnitine profile in plasma, in order to diagnose deficiencies connected with metabolism of fatty acid oxidation disorders and organic acidemias, is possible with the application of LC-MS/MS [9]. The simultaneous analysis of amino acids and acylcarnitine panels with LC-MS/MS provides information on about 40 metabolic disorders such as phenylketonuria (PKU) and medium chain Acyl-CoA Dehydrogenase Deficiency (MCAD). A simple flow-injection analysis gives results in 2 minutes with minimal sample preparation. Researchers have recently extended the applicability of LC-MS/MS for determination of a very long chain of fatty acids for characterizing Peroxisomal Disorders and other clinically relevant markers such as the panel 17-OH progesterone/Androstenedione/Cortisol for characterizing Congenital Adrenal Hyperplasia (CAH) [10].

Capillary electrophoresis - electrospray ionization-mass spectrometry (CE-ESI-MS), applied first in 2009, offers a convenient platform for the direct analysis of amino acids, acylcarnitines, and their stereoisomers from dried blood spot (DBS) extracts without chemical derivatization [11].

\section{Coupled TeChNiques in Other CuRrent Medical DiaGNOSES OF DISEASES}

The coupling gas chromatography with mass spectrometry is used to determine homovanillic acid (HVA), (dopamine metabolite) and vanilmandelic acid (VMA) (adrenaline, noradrenaline metabolites) in urine of patients with Costello syndrome, which is a genetic disorder that affects many parts of the body, characterized by delayed development and mental retardation, distinctive facial features, loose folds of extra skin (especially on the hands and feet), unusually flexible joints, and higher risk of cancers including neuroblastoma [12]. The level of HVA acid is also very essential in the case of people with neurological disorders such as autism and some other related disorders $[13,14]$. Another mental retardation syndrome associated with deficient cholesterol synthesis is Smith-Lemli-Opitz syndrome (SLO), caused by inactivity of the enzyme 7-dehydrosterol$\Delta^{7}$-reductase, which catalyses the terminal transformation in cholesterol synthesis. An increased level of 7-dehydrocholesterol in blood and tissues of patients, and also an increased level of 8-dehydrocholesterol, were found to be due to the presence of an active $\Delta^{8}-\Delta^{7}$ isomerase. A major consequence of these biochemical abnormalities is the alteration of normal embryonic and fetal somatic development, resulting in postnatal abnormalities of growth, learning, language and behavior. SLO syndrome is easily recognized with the application of GC-MS technique [15].

Coupled chromatographic techniques based on mass spectrometry are successfully used in the diagnosis of digestive system diseases. A method for the separation and simultaneous determination of urinary D- and L-lactic acid enantiomers by (HPLC-MS/MS) is recommended. A higher level of D-lactic acid found in human urine often proves the presence of bacteria in the human gastrointestinal tract or intestines [16]. LC-MS/MS is also a technique convenient 
for the determination of 17 alpha-hydroxyprogesterone, and enables early and higher determination of levels of congenital adrenal hyperplasia (CAH) markers. This disease may lead to death, and cure is possible only with an early diagnosis. Sensitivity and specificity of LC-MS/MS give more accurate results than when conventional biochemical investigations are made [17].

Colon cancer is one of the main causes of death worldwide. Thus, it is important to find a new prognostic and diagnostic marker of this disease, as well as to elucidate the special metabolic pathways of colon cancer cells. Zimmermann and co-workers were the first to determine some qualitative differences in the profiles of the volatile metabolites of colon cancer cell lines among themselves and in comparison with the normal colon cell line. The discrepancies were connected with ketones and alcohols, which were determined by applying solid phase microextraction (SPME) and GC-MS methods. For the first time, the compounds like undecan2-ol and pentadecan-2-one were associated with the human metabolism. These findings indicate that the metabolism of colon cancer cells differs extremely from the metabolism of healthy cells, and it changes during the progress of the disease [18].

GC-MS have also made it possible to determine volatile biomarkers of pulmonary tuberculosis in human breath. The identified compounds are as follows: naphthalene, 1-methyl-, 3-heptanone, methylcyclododecane, heptane, 2,2,4,6,6-pentamethyl-, benzene, 1-methyl-4-(1-methylethyl)-and cyclohexane, 1,4-dimethyl. The volatile biomarkers determined in breath were sensitive and specific for pulmonary tuberculosis - the breath test distinguished between "sick versus well" (i.e., between normal controls and patients hospitalized due to suspicion of pulmonary tuberculosis), and between infected versus non-infected patients (i.e., between those whose sputum cultures were positive or negative for Mycobacteria) [19].

Coupled techniques are also applied in the diagnosis of cardiovascular diseases. GC-MS allows measuring the level of isoprene, reputed to reflect oxidative stress, which plays a critical role in chronic heart failure. Oxidative stress plays a crucial role in carotid atherosclerosis. To determine markers of oxidative stress, the contents of erythrocyte membrane lipids in the case of people with Type 2 diabetes were analyzed [20]. 8-Hydroxy-2'-deoxyguanosine (8OHdG) is considered to be an excellent marker of oxidative DNA damage. Studies showed that urinary $8-\mathrm{OHdG}$ is a good biomarker for risk assessment of various cancers such as breast cancer [21], and degenerative diseases. Both GC/MS and LC/MS are widely used in the detection of 8-OHdG. The biomarker has been used to estimate the DNA damage in humans after exposure to cancer-causing agents such as tobacco smoke, asbestos fibers, heavy metals, and polycyclic aromatic hydrocarbons [22]. The National Institute of Environmental Health Sciences (NIEHS) has a mission to reduce the burden of human illness and disability by understanding how the environment influences the development and progression of human disease, and has established an initiative to conduct a comparative study of biomarkers of oxidative stress (BOSS) in order to find out whether a fingerprint for measurement of oxidative stress exists. To evaluate the available methodology for measuring oxidative stress with potential application to human studies, NIEHS has taken the lead in organizing the first international multi-laboratory comprehensive comparative study for determining which of the available biomarkers of oxidative stress are the most specific, sensitive and selective. The aim of BOSS is to determine measurable, sensitive, and specific biomarkers for oxidative damage in rodents, non-human primates, and humans, resulting from multiple types of oxidative insults and to understand the relationships among markers, which will be useful for choosing an appropriate marker in a given study [23].

Isoprostanes are derivatives of 1 specific lipid - arachidonic acid - after lipid peroxidation. Several isoprostanes have been identified in biological tissues and fluids, including 8-iso prostaglandin F2alpha (8-iso-PGF2alpha, 8-epi-PGF2alpha, iPF2alpha-III, 15-F2t-IsoP) and its metabolite, 2,3-dinor-4,5-dihydro-8-iso-PGF2alpha. Isoprostanes are reliable in vivo markers of lipid peroxidation in humans. The determination of isoprostanes using HPLC, LC-MS/MS, GC-MS and GC-MS/MS can be exemplified in the field of cardiovascular research. The results of studies indicate that isoprostanes are surrogate parameters of oxidative stress in cardiovascular disease [24,25]. Other research [26] suggests that the amount of carbonyl groups and 3-nitrotyrosine in plasma proteins may be important indicators of protein damage in vivo in schizophrenia.

Mounting evidence suggests the role of oxidative stress in the development and clinical manifestation of autism. There is evidence of the disruption of antioxidant defense mechanisms in autism, manifested by lower than control levels of glutathione peroxidase (GSPHx), as evidenced by lower levels of plasma glutathione and higher ratios of oxidized glutathione to reduced glutathione. 8 -Hydroxyguanine (8-OHG) is one of the oxidative stress urine markers of RNA oxidation in mitochondria. Monitoring of plasma glutathione levels and 8 -Hydroxyguanine in urine of autistic children is possible with an application of 2 chromatographic methods: GC-MS and LC-MS [27,28].

Alzheimer's disease (AD) is a neurodegenerative disorder, pathologically characterized by the progressive deposit of a specific form of amyloid and amyloid- $\beta$ peptides (A $\beta$ ) (marker Alzheimer's disease) in the brain. LC-MS and CE-MS coupled with MS are used to find the specific form of amyloid in plasma. Capillary electrophoresis (CE) coupled with mass spectrometry (MS) is a powerful separation technique, but its low sensitivity limits its use in the analysis of biological matrices. Thus, LC-MS is a more suitable technique in monitoring of amyloid- $\beta$ peptides $(A \beta)$ [29].

\section{Coupled Techniques in Cancer Therapies}

Chromatographic techniques coupled with MS can be applied in monitoring of cancer therapies. LC-MS/MS method was developed for the evaluation of motexafin gadolinium (MGd) pharmacokinetics and biodistribution in plasma and tissues. Motexafin gadolinium is a synthetic, pentadentate, aromatic Gd-containing macrocyclic complex used in treatments of children's brain tumors and pancreatic cancer. The method, which shows a fast runtime and high selectivity, was used for routine clinical plasma sample analysis [30]. Vinorelbine is a semi-synthetic vinca alkaloid compound, 
which was introduced as an antineoplastic agent. The application of the agent in an oral form requires information about its metabolic pathway, and in consequence, higher sensitivity and specificity of the analytical method. Liquid chromatography method coupled with tandem mass spectrometric detection appears to be best in the clinical analysis of vinorelbine and its metabolites in blood, plasma, urine and faces [31].

Boron neutron capture therapy (BNCT) is a promising binary treatment for cancer, based on nuclear fission that occurs when non-radioactive ${ }^{10} \mathrm{~B}$ absorbs thermal neutrons. One of the 2 boron compounds currently used in clinical trials for this therapy is BSH (closo-undeca- hydro-1-mercaptododecaborate) disodium salt. To ensure differentiated retention in the tumor versus normal tissue prior to treatment, routine analytical methods of pharmacokinetics determination must be available. For this purpose, CE-ESI-MS method was used in separation of boron derivatives. In comparison with other techniques, it has some advantages - simple sample preparation, high selectivity and negligible matrix interference, high sensitivity and short analysis time [32].

\section{CoUPLed TeChNiques in the InVESTIGATION OF NeW DRUGS}

The World Health Organization (WHO) estimated that in 2009 about 132 million people in developed countries lived with diabetes, and it is estimated that in 2025227 million people will have this disease [33]. Thus, there is great social and economic need for an adequate treatment. R483, a new insulin sensitizer, belongs to the thiazolidinediones (or glitazones), representing a new class of orally active drugs that reduce insulin resistance. In order to develop a racemic drug, the pharmacological effects of each enantiomer should be evaluated, because different enantiomers can have different pharmacodynamic effects. In the best case, one of the enantiomers has an equal or less pronounced effect than the other, and in the worst case it is toxic. Chiral HPLC with electrospray tandem mass spectrometric detection seems to be the best method for the determination of the enantiomeric composition of a new insulin sensitizer in plasma samples [34]. The plasma concentration of olmesar$\tan$ (the prodrug of olmesartan medoxomil) was determined using high-performance liquid chromatography coupled with electrospray tandem mass spectrometry (LC-MS/MS).

Olmesartan medoxomil is a drug used in the treatment of high blood pressure (hypertension), and is a type of a drug called an angiotensin II receptor blocker. It may be used alone or with other drugs in the treatment of high blood pressure. The LC-MS/MS method was successfully applied in a bioequivalence study of an olmesartan formulation after administration as a single oral dose [35].

LC-MS/MS is a simple, sensitive and highly selective method used in the therapy of allergic diseases for simultaneous identification of pseudoephedrine and cetirizine in human plasma. The advantage of this method is the minimal sample preparation, as the procedure is only one-step protein precipitation [36]. A simple, sensitive and highly selective liquid chromatography/tandem mass spectrometry (LC-MS/MS) method was developed to simultaneously determine the concentrations of pseudoephedrine and cetirizine in human plasma.
A simple analytical method was developed in plasma for the simultaneous assay of the 7 nucleoside/nucleotide reverse transcriptase inhibitors (abacavir, didanosine, emtricitabine, lamivudine, stavudine, tenofovir, and zidovudine) currently used for the treatment of HIV-infected patients. The method has been used to assess plasma concentrations in HIV-infected patients and was found to be suitable for therapeutic drug monitoring [37].

Zolmitriptan is a novel 5- $\mathrm{HT}_{1 \mathrm{~B} / 1 \mathrm{D}}$ receptor agonist developed for the acute oral treatment of migraine. A highly sensitive LC-MS/MS assay has been developed for quantification of plasma concentrations in the case of zolmitriptan and its active metabolite $-\mathrm{N}$-desmethylzolmitriptan. The validated method can be applied in pharmacokinetic and bioequivalence studies [38]. Shortage of vitamin K causes a decrease in mineral density and an increase in bone resorption. GC-MS and LC-MS are among the important determination methods of the pharmacokinetics and pharmacodynamics of vitamin K capsules in human plasma [39].

\section{COUPLED TECHNIQUES IN ENVIRONMENTAL RISK EVALUATION AND BIOMONITORING}

Biological fluids such as urine, plasma, cerebrospinal fluid and tissue are great matrices for the clinical investigations of the environmental risk evaluation in which chromatographic techniques coupled with mass spectrometry are used. A new method based on liquid chromatography tandem mass spectrometry was developed for the direct determination of nicotine, cotinine, trans-3'-hydroxycotinine, their corresponding glucuronide conjugates as well as nornicotine, norcotinine, cotinine- $N$-oxide and nicotine- $N$-oxide in the urine of smokers. The high range of limit detection and high sensitivity of this method make it possible to examine the nicotine dose in large-scale human biomonitoring studies [40].

Manganese (Mn) at high concentrations can have adverse effects on health, mainly because of its toxicity to the central nervous system, resulting Parkinson's disease. Health impacts of Mn are known mostly from occupational health studies, but the exact mechanisms of how Mn being bound to transferrin (TF) in the blood enters the brain are unknown. Mn speciation at the neural barriers can help to obtain more information about the pathways and carriers [41].

Urinary trans, trans-muconic acid ( $t, t$-MA) is a biomarker of benzene exposure. Usually, a biomarker of benzene exposure was determined by LC-UV methods, but recently LC-MS/MS method with isotopic dilution for quantitative determination of trans, trans-muconic acid in urine samples of workers exposed to low benzene concentrations was developed. This method makes it possible to distinguish samples coming from different environmental high risk groups including cigarette smokers and their different combinations [42]. Tyrosine metabolism disorders may be the result of inborn errors metabolism and they can also be caused by the activity of xenobiotics. Dichloroacetate (DCA) is a part of environmental pollution, as it is a by-product of water chlorination and industrial degreasing agents (e.g., trichloroethylene). In certain diseases, it may be used as a drug. A GC-MS/MS method was used in the quantitative analysis of low levels of the tyrosine metabolites maleylacetone, 


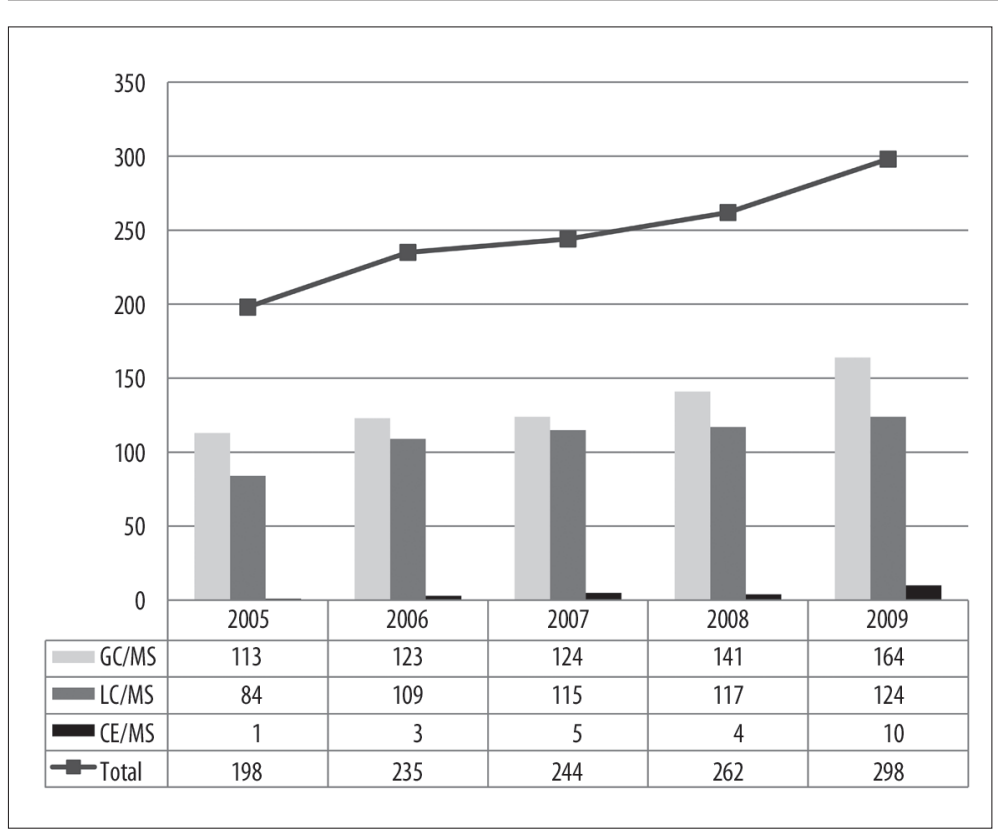

Figure 1. Bibliographic search in chemical Abstract with the key words GC-MS or LC-MS or CE-MS and in current medical research using Sci Finder Scholar up to December $9^{\text {th }}, 2009$.

succinylacetone, and the tyrosine metabolism dichloroacetate inhibitor in biological fluids and tissues [43].

\section{CONCLUSIONS}

The coupled techniques based on chromatography, spectrometry and related techniques are widely used in different medical analyses. Coupling of these techniques improves their specificity and sensitivity. They allow performing a fast and simple analysis, and in most cases do not require any preliminary preparation of samples. They are more reliable and suitable to determine trace concentrations of substances present in biological fluids than are classical biochemical examinations.

An increasing interest is anticipated in the near future in chromatographic methods coupled with spectrometry in medical research is not only connected with high resolution but it also improves the limit of detection and potential biomarker identification. Figure 1 shows the frequency of total medical studies with application of GC-MS, LC-MS, and CE-MS methods in the period from 2005 to 2009.

\section{REFERENCES:}

1. Hübschmann HJ: Handbook of GC/MS: Fundamentals and Applications Germany, Weinheim: Wiley-VCH, 2009

2. Wu Z, Huang Z, Lehmann R et al: The application of chromatographymass spectrometry: methods to metabolomics. Chromatographia, 2009; 69: 23-32

3. Dunn WB, Ellis DI: Metabolomics: Current analytical platforms and methodologies. Trends Anal Chem, 2005; 24: 285-94

4. Mayboroda OA, NeusuB Ch, Pelzing M et al: Amino acid profilling in urine by capillary zone electrophoresis-mass spectrometry. J Chromatogr A, 2007; 1159: 149-53

5. Song YZ, Li BX, Hao H et al: Selective screening for inborn errors of metabolism and secondary methylmalonic aciduria in pregnancy at high risk district of neural tube defects: a human metabolome study by GCMS in China. Clin Biochem, 2008; 41 (7-8): 616-20

6. Kuhara T: Noninvasive human metabolome analysis for differential diagnosis of inborn errors of metabolism. J Chromatogr B. 2007; 855(1): $42-50$
7. Hasagawa Y, Iga M, Kimura M et al: Prenatal diagnosis for organic acid disorders using two mass spectrometric methods, gas chromatography mass spectrometry and tandem mass spectrometry. J Chromatogr B, 2005; 823: 13-17

8. Rao AN, Kavitha J, Koch M, Kumar SV: Inborn errors of metabolism: Review and data from a tertiary care center. Indian J Clin Bioch, 2009; 24(3): 215-22

9. Ferrer I, Ruiz-Sala P, Vincente Y et al: Separation and identification of plasma short-chain acylocarnitine isomers by HPLC/MS/MS for the differential diagnosis of fatty acid oxidation defects and organic acidemias. J Chromatogr B, 2007; 860: 121-26

10. The $6^{\text {th }}$ ISNS European Regional Meeting in Neonantal Screening, Prague, Czech Republik, April 26-28 ${ }^{\text {th }}$ 2009. Neonantal Screening, the Europen Perspective. Ces Slov Pediat, 2009; 64

11. Chalcraft KR, Britz-McKibbin P: Newborn Screening of Inborn Errors of Metabolism by Capillary Electrophoresis - Electrospray IonizationMass Spectrometry: A Second-Tier Method with Improved Specificity and Sensitivity. Anal Chem, 2009; 81(1): 307-14

12. Bowron A, Scott JG, Brewer C, Weir P: Increased HVA detected on organic acid analysis in a patient with Costello syndrome. J Inherit Metab Dis, 2005; 28: 1155-56

13. Socha E, Michalska M, Kałużna-Czaplińska J, Rynkowski J: GC/MS for screening of dopamine disorders marker in urine of autistic children. Acta Biochim, 2008; 55: S3-31

14. Kałużna-Czaplińska J, Socha E, Michalska M et al: The level of homovanillic acid in urine of autistic children. Medycyna Rodzinna, 2009; 2 48-52

15. Witsch-Baumgartner M: DHCR7 mutations causing the Smith-LemliOpitz syndrome. Future Lipidology, 2008; 3: 585-93

16. Norton D, Crow B, Bishop M et al: High performance liquid chromatography-tandem mass spectrometry (HPLC/MS/MS) assay for chiral separation of lactic acid enantiomers in urine using a teicoplanin based stationary phase. J Chromatogr B, 2007; 850: 190-98

17. Elter ML, Eichhorst J, Lehotay DC: Clinical determination of 17-hydroxyprogesterone in serum by LC-MS/MS: Comparison to Coat-ACount $^{\mathrm{TM}}$ RIA method. J Chromatogr B, 2006; 840: 69-74

18. Zimmermann D, Hartmann M, Mayer MP et al: Determination of volatile products of human colon cell line metabolism by GC/MS analysis. Metabolomics, 2007; 3: 13-17

19. Philips M, Cataneo RN, Condos R et al: Volatile biomarkers of pulmonary tuberculosis in the breath. Tuberculosis, 2007; 87: 44-52

20. King A, Priesbe T: Relationship between carotid atherosclerosis and erythrocyte membrane cholesterol oxidation products in type 2 diabetic patients. Diab Res Clin Pract, 2009; 72(3): 331-34

21. Kuo HW, Chou SY, Hu TW et al: Urinary 8-hydroxy-2'-deoxyguanosine (8-OHdG) and genetic polymorphisms in breast cancer patients. Mutat Res, 2007; 631(1): 62-68 
22. Mei S, Yao Q, Wu C, Xu G: Determination of urinary 8-hydroxy-2'-deoxyguanosine by two approaches-capillary electrophoresis and GC/ MS: An assay for in vivo oxidative DNA damage in cancer patients. J Chromatogr B, 2005; 827: 83-87

23. http://www.niehs.nih.gov

24. Schwedhelm E, Böger RH: Application of gas chromatography-mass spectrometry for analysis of isoprostanes: their role in cardiovascular disease. Clin Chem Lab Med, 2003; 41(12): 1552-61

25. Yin H: New techniques to detect oxidative stress markers: mass spectrometry-based methods to detect isoprostanes as the gold standard for oxidative stress in vivo. Biofactors, 2008; 34(2): 109-24

26. Dietrich-Muszalska A, Olas B, Głowacki R, Bald E: Oxidative/nitrative modifications of plasma proteins and thiols from patients with schizophrenia. Neuropsychobiology, 2009; 59(1): 1-7

27. Rao AN, Koch M, Kumar SV et al: Assessment of Metabolic Parameters For Autism Spectrum. OJHAS, 2009; 8(1)

28. Kałużna-Czaplińska J, Grys W, Rynkowski J, Szymańska A: Role of oxidative stress in autism. Nowa Pediatria, 2008; 4: 67-70

29. Zhang X, Rauch A, Xiao H et al: Mass spectrometry-based neurochemical analysis: perspectives for primate research. Exp Rev Proteom, 2008 V5(5): 641-52

30. Miles DR, Mesfin M, Mody TD et al: Validation and use of three complementary analytical methods (LC-FLC, LC-MS/MS and ICP-MS) to evaluate the pharmacokinetics, biodistribution and stability of motexafin gadolinium in plasma and tissues. Anal Bioanal Chem, 2006; 385 : 345-56

31. Puozzo C, Ung HL, Zorza G: High performance liquid chromatography method for vinorelbine and 4-O-deacetyl vinorelbine: A decade of routine analysis in human blood. J Pharm Biom Anal, 2007; 44(1): 144-49

32. Pitois A, de las Heras LA, Zampolli A et al: Capillary electrophoresiselectrospray mass spectrometry and HR-ICP-MS for the detection and quantification of 10B-boronophenylalanine (10B-BPA) used in boron neutron capture therapy. Anal Bioanal Chem, 2006; 384: 751-60
33. http://www.who.int/en/

34. Lausecker B, Fischer G: Determination of the enantiomeric composition of a new insulin sensitizer in plasma samples from non-clinical and clinical investigation using chiral HPLC with electrospray tandem mass spectrometric detection. J Chromatogr B, 2006; 835: 40-46

35. Vaidya VV, Roy SMN, Yetal SM et al: LC-MS-MS determination of olmesartan in human plasma. Chromatographia, 2008; 67: 147-50

36. Ming M, Fang F, Yulan S et al: Development and evaluation of an efficient HPLC/MS/MS method for the simultaneous determination of pseudoephedrine and cetirizine in human plasma: Application to Phase-I pharmacokinetic study. J Chromatogr B, 2007; 846: 105-11

37. Le Saux T, Chhun S, Rey E et al: Quantification of seven nucleoside/ nucleotide reverse transcriptase inhibitors in human plasma by highperformance liquid chromatography with tandem mass-spectrometry. J Chromatogr B, 2008; 865: 81-90

38. Kilic B, Ozden T, Toptan S, Ozilhan S: Simultaneous LC-MS-MS determination of zolmitriptan and its active metabolite N-Desmethylzolmitriptan in human plasma. Chromatographia, 2007; 66: 129-33

39. Song O, Wen A, Ding L et al: HPLC-APCI-MS for the determination of vitamin $\mathrm{Kl}$ in human plasma: Method and clinical application. J Chromatogr B, 2008; 875: 541-45

40. Fan Z, Xie F, Xia Q et al: Simultaneous determination of nicotine and its nine metabolites in human urine by LC-MS-MS. Chromatographia, 2008; 68: 623-27

41. Finley JW: Does Environmental Exposure to Manganese Pose a Health Risk to Healthy Adults? Nutrition Reviews, 2004; 62(4): 148-53

42. Tranfo G, Paci E, Sisto R, Pigini D: Validation of an HPLC/MS/MS method with isotopic dilution for quantitative determination of trans, trans-muconic acid in urine samples of workers exposed to low benzene concentrations. J Chromatogr B, 2008; 867: 26-31

43. Zolodz MD, Jia M, Liu H et al: A GC-MS/MS method for the quantitative analysis of low levels of the tyrosine metabolites maleylacetone, succinylacetone, and the tyrosine metabolism inhibitor dichloroacetate in biological fluids and tissues. J Chromatogr B, 2006; 837: 125-32 